\title{
Suboptimal Dosing Parameters as Possible Factors in the Negative Phase III Clinical Trials of Progesterone for Traumatic Brain Injury
}

\author{
Randy B. Howard, Iqbal Sayeed, ${ }^{2}$ and Donald G. Stein ${ }^{2}$
}

\begin{abstract}
To date, outcomes for all Phase III clinical trials for traumatic brain injury (TBI) have been negative. The recent disappointing results of the Progesterone for the Treatment of Traumatic Brain Injury (ProTECT) and Study of a Neuroprotective Agent, Progesterone, in Severe Traumatic Brain Injury (SyNAPSe) Phase III trials for progesterone in TBI have triggered considerable speculation about the reasons for the negative outcomes of these two studies in particular and for those of all previous Phase III TBI clinical trials in general. Among the factors proposed to explain the ProTECT III and SyNAPSe results, the investigators themselves and others have cited: 1) the pathophysiological complexity of TBI itself; 2) issues with the quality and clinical relevance of the preclinical animal models; 3) insufficiently sensitive clinical endpoints; and 4) inappropriate clinical trial designs and strategies. This paper highlights three critical trial design factors that may have contributed substantially to the negative outcomes: 1) suboptimal doses and treatment durations in the Phase II studies; 2) the strategic decision not to perform Phase IIB studies to optimize these variables before initiating Phase III; and 3) the lack of incorporation of the preclinical and Chinese Phase II results, as well as allometric scaling principles, into the Phase III designs. Given these circumstances and the exceptional pleiotropic potential of progesterone as a TBI (and stroke) therapeutic, we are advocating a return to Phase IIB testing. We advocate the incorporation of dose and schedule optimization focused on lower doses and a longer duration of treatment, combined with the addressing of other potential trial design problems raised by the authors in the recently published trial results.
\end{abstract}

Key words: allometric scaling; Phase III trials; progesterone; suboptimal dosing

\section{Introduction}

A LTHOUGH THE NEUROSTEROID PROGESTERONE SHOWED considerable preclinical efficacy as a neuroprotective agent in the treatment of traumatic brain injury (TBI), and despite two moderately encouraging Phase II clinical reports, two similarly designed Phase III TBI clinical trials recently resulted in negative outcomes. ${ }^{1,2}$ Reporting the lack of significant differences between treatment and control groups in the Progesterone for the Treatment of Traumatic Brain Injury (ProTECT) III trial, the authors write that "it is possible that the heterogeneity of the injury, confounding preexisting conditions, and characteristics of individual patients (e.g., resilience) which can be well controlled in animal studies, play too large a role to overcome in human disease." ${ }^{1}$ The authors of the similarly negative Study of a Neuroprotective Agent, Progesterone, in Severe Traumatic Brain Injury (SyNAPSe) trial ${ }^{2}$ speculate that the outcome "is probably due to several factors, including the complexity and variability of the injury and the fact that multiple direct and indirect injury mechanisms are at work simultaneously." They also cite insensitivity of the outcome measures and characterization of the complexity of TBI as factors that need to be corrected to advance therapeutics in this area.

Although all these factors may have contributed to the disappointing final outcomes, three so far unaddressed and potentially critical additional issues are: 1) the possibility that the doses and treatment durations used in Phase II were suboptimal; 2) the fact that these Phase II parameters were not then optimized in further Phase IIB studies prior to initiating Phase III; and 3) the fact that the two very similar Phase III studies were then designed without taking into account new preclinical reports, the Chinese Phase II clinical results, and appropriate allometric scaling considerations needed to translate preclinical laboratory findings to human patients.

\footnotetext{
${ }^{1}$ Pharmacology Consultant, Drug Discovery and Development, ${ }^{2}$ Department of Emergency Medicine, Emory University, Atlanta, Georgia.

(C) Randy B. Howard, Iqbal Sayeed, Donald G. Stein, 2015; Published by Mary Ann Liebert, Inc. This Open Access article is distributed under the terms of the Creative Commons Attribution Noncommercial License (http://creativecommons.org/licenses/by-nc/4.0/) which permits any noncommercial use, distribution, and reproduction in any medium, provided the original author(s) and the source are credited.
} 
Based on our evaluation of the preclinical findings and all four clinical reports, ${ }^{1-4}$ we propose that the negative outcomes in Phase III may well have been primarily due to the use of too high a drug dose and too short a period of administration.

\section{Overview of the Phase II Clinical and Preclinical Reports}

The Phase II studies were ProTECT II, done in a single Level 1 trauma center in Atlanta, GA, beginning in $2001{ }^{3}$ and a second Level 1 trauma center study in Hangzhou, China, which began in $2004 .{ }^{4}$ The ProTECT II trial design and resources allowed for just two groups: intra-lipid vehicle and progesterone. The latter was provided at a dose of $12 \mathrm{mg} / \mathrm{kg} /$ day for 3 days - a relatively high but safe dose. Drug and vehicle were given by a series of six 12-hour intravenous infusions at $0.5 \mathrm{mg} / \mathrm{kg} / \mathrm{h}$. A total of 100 patients with Glasgow Coma Scale (GCS) scores of 4 to 12 (with higher scores indicating less injury) were admitted to the study and randomized 4:1 in treated versus vehicle groups. The main outcomes measured were mortality, and Glasgow Outcome Scale (GOS), Extended GOS (GOS-E), and Disability Rating Scale (DRS) scores, with the patients stratified into severely injured (GCS 4-8) and moderately injured (GCS 9-12) subgroups. Patients were evaluated for 1 month post-injury only. The results indicated that the treated patients had significantly reduced mortality at 30 days; however, the severely injured group did not reflect any GOS-E or DRS improvements, compared with the controls. In contrast, the relatively small, moderately-injured subgroup of 25 patients reportedly showed significantly improved GOS-E and DRS scores with treatment.

In the Chinese Phase II trial (retrospectively registered in the Australian New Zealand Clinical Trials Registry), ${ }^{4}$ a much lower dose was chosen, apparently by doubling what was thought to be a $1 \mathrm{mg} / \mathrm{kg} /$ day dose being used in the lead ProTECT II study, to $2 \mathrm{mg} /$ $\mathrm{kg} / \mathrm{day} .{ }^{4}$ Dosing was given by intramuscular injection b.i.d. in camellia oil for 5 days. A total of 159 patients with GCS scores of 3-8 (i.e., all severely injured) were randomized 1:1. The main outcomes measured included mortality, regular GOS scores, and Functional Independence Measure (FIM) scores. In contrast to ProTECT II, the results were reported later, at 3 and 6 months. As reported for severely-injured patients in ProTECT II, patients in this trial had reduced mortality, but importantly they also had improved GOS scores at both 3 and 6 months, unlike the severely-injured ProTECT II patients evaluated at 1 month. While it has been noted that the positive GOS effect at 6 months had dropped to only borderline significance $(p=0.048),{ }^{5}$ there were 159 patients, lending credence to a functional efficacy signal in these severely-injured patients with this much lower dose and slightly longer schedule $(2 \mathrm{mg} / \mathrm{kg} / \mathrm{day} \times 5$ days $)$.

The dosing approach that was taken in the lead ProTECT II study implies two assumptions-first, that the TBI progesterone doseresponse curve would be of the conventional sigmoidal type and that therefore using a high $12 \mathrm{mg} / \mathrm{kg} /$ day progesterone dose would likely enable demonstration of maximal efficacy with just one treated group. This dose resulted in progesterone plasma drug levels (PDLs) of $337 \pm 135 \mathrm{ng} / \mathrm{mL}$ in TBI patients, ${ }^{6}$ which is much higher than the PDLs well below $100 \mathrm{ng} / \mathrm{mL}$ that were measured in a rat pharmacokinetic study associated with a dose that reduced TBI edema by $37 \% .^{7}$ The second assumption was that the 3-day treatment duration used in laboratory rats was long enough to allow demonstration of significant neuroprotective efficacy in humans. However, based on subsequent preclinical dose-response and dose-duration studies, and consideration of interspecies allometric dose scaling and relative brain healing times for humans and rats, it appears likely that neither assumption was valid.
After ProTECT II had already begun, rat preclinical reports appeared demonstrating that 5 days of treatment were more effective than 3 days in reducing edema, local necrosis, and distant cell loss in thalamic nuclei, and that at least 5 days of treatment was needed to see improved functional outcomes in spatial learning performance and sensory neglect assays. ${ }^{8,9}$ An additional study then showed that rat doses of 8 and $16 \mathrm{mg} / \mathrm{kg} / \mathrm{day}$ for 5 days resulted in similarly improved spatial learning in the Morris water maze and reduced sensory neglect. But at a higher dose of $32 \mathrm{mg} / \mathrm{kg} /$ day, efficacy was either reduced or entirely lost. ${ }^{10}$ These pre-clinical results were interpreted to indicate that these functional endpoints actually showed a bell-shaped dose-response relationship, revealing that too high a dose resulted in a loss of functional efficacy-a finding with an important implication for selecting optimal doses for clinical use.

More recent and extensive experiments in rat stroke models ${ }^{11,12}$ also are consistent with bell-shaped curves for progesteronemediated neuroprotection, where $32 \mathrm{mg} / \mathrm{kg}$ for 7 days resulted in reduced progesterone efficacy compared with 8 and $/$ or $16 \mathrm{mg} / \mathrm{kg}$, for a variety of functional outcomes including locomotor activity, grip strength, sensory neglect, gait impairment, motor coordination, and spatial navigation. It also may be worth noting that while earlier rat stroke studies had shown efficacy with single $4 \mathrm{mg} / \mathrm{kg}$ doses given immediately prior to ischemia, high single doses of 30 or $60 \mathrm{mg} / \mathrm{kg}$ were ineffective, and chronic prior dosing at $30 \mathrm{mg} / \mathrm{kg}$ for 7-10 days actually increased infarction volume. ${ }^{13}$

A loss of effectiveness at high doses is not unique to progesterone use for neuroprotection. Bell-shaped dose-response characteristics also occur in drugs developed to block tumor angiogenesis in cancer ${ }^{14}$ and with the use of superoxide dismutases (SODs) in the treatment of ischemic reperfusion injury to heart, brain, and intestine. ${ }^{15}$ In particular, the latter paper notes "the consistent loss of protection at higher doses of SOD, even though the models differ considerably with respect to ... species, ... end point measured, ... source of the SOD used .... and its schedule of administration ...."

The preclinical results reviewed here have potentially important implications for human clinical trials with progesterone. First, they suggest that the $12 \mathrm{mg} / \mathrm{kg}$ /day human dose used in ProTECT II and both Phase III trials may have been too high, possibly resulting in the same reduced or lost effectiveness seen in the animal studies at the $32 \mathrm{mg} / \mathrm{kg}$ dose. To see why this is the case, one needs to convert the rat $\mathrm{mg} / \mathrm{kg} / \mathrm{d}$ dose to the human dose using allometric scaling. To convert rat $\mathrm{mg} / \mathrm{kg}$ doses to human $\mathrm{mg} / \mathrm{kg}$ doses for compounds like progesterone that are excreted primarily by the kidneys and have relatively uncomplicated metabolic behaviors, the most accurate formula ${ }^{16}$ requires multiplying the rat $\mathrm{mg} / \mathrm{kg}$ dose by the conversion factor of (Wt rat/Wt human $)^{0.25}$. For a $0.3 \mathrm{~kg}$ rat and an $80 \mathrm{~kg}$ human, the above formula works out to $(0.00375)^{0.25}=0.25$. Multiplying the Goss study rat doses of 8,16 , and $32 \mathrm{mg} / \mathrm{kg} / \mathrm{day}$ by 0.25 gives "equivalent" scaled human doses of 2,4 and $8 \mathrm{mg} / \mathrm{kg} / \mathrm{day}$. Combined with the rat efficacy data above, this result suggests that human progesterone efficacy in TBI might be expected to begin to drop at a dose somewhere between 4 and $8 \mathrm{mg} / \mathrm{kg} /$ day, which is considerably below the $12 \mathrm{mg} / \mathrm{kg} /$ day used in ProTECT II and both Phase III trials. Conversely, it suggests that human doses roughly in the 2 to $4 \mathrm{mg} / \mathrm{kg}$ / day range, like those used in the Chinese Phase II study, are more likely to be effective. While our estimates suggest that doses below $8 \mathrm{mg} / \mathrm{kg} /$ day may be more effective in humans, only an empiricallybased dose optimization in a Phase IIB trial under defined conditions (vehicle, route, dose, and schedule) can determine what dose or doses are optimal (Fig. $1^{2,6,7,17-19}$ ). 


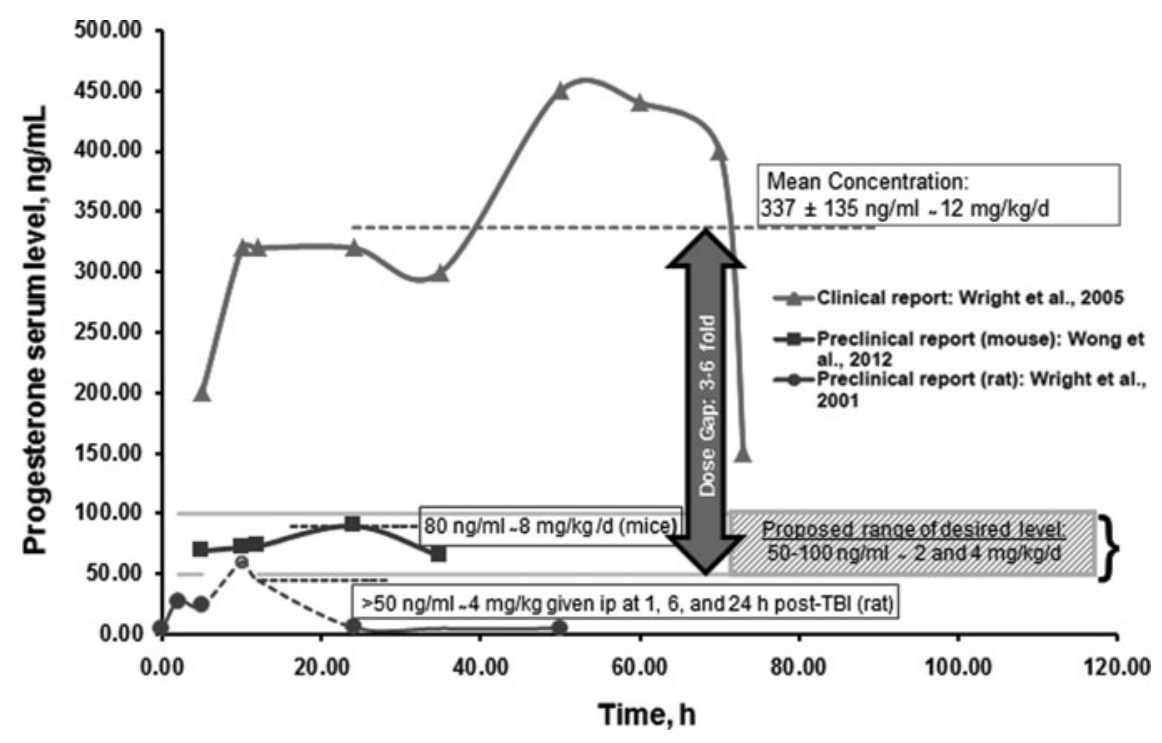

FIG. 1. Hypothetical representation of optimum progesterone (PROG) serum levels needed for functional recovery in future clinical trials: The figure illustrates serum progesterone levels in: 1) a published clinical trial; 2) two preclinical papers; and 3) our proposed hypothetical range of desired PROG serum levels. The clinical trajectory is based on Wright and colleagues, ${ }^{6}$ which reports the steadystate serum concentration of PROG $(337 \mathrm{ng} / \mathrm{mL})$ following continuous intravenous infusion in patients with acute moderate to severe traumatic brain injury (TBI). The dosing is comparable to the median serum level $(335 \mathrm{ng} / \mathrm{mL}, 2$ days after initial dose) used in the SyNAPSe clinical trial. ${ }^{2}$ The trajectories for the preclinical dosing are representative of two studies: a pharmacokinetic report showing PROG concentration in mice treated with $8 \mathrm{mg} / \mathrm{kg} / \mathrm{d}$ of PROG delivered by implanted osmotic minipumps (simulating continuous

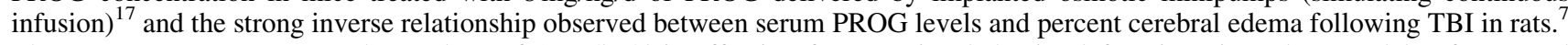
There are numerous reports that a dose of $8 \mathrm{mg} / \mathrm{kg} / \mathrm{d}$ is effective for restoring behavioral functions in rodent models of TBI and stroke. ${ }^{18,19}$ Our hypothetical optimal serum level range of $50-100 \mathrm{ng} / \mathrm{mL}$ ( 2 and $4 \mathrm{mg} / \mathrm{kg}$ doses for a proposed new Phase IIB trial) is based on an allometric calculation as follows: $1 / 6$ and $1 / 3$ of the $12 \mathrm{mg} / \mathrm{kg}$ dose used in the recent clinical trial reports corresponding to human plasma levels of $\sim 50(335 / 6)$ and $\sim 100(335 / 3) \mathrm{ng} / \mathrm{mL})$. The data points in this figure are estimated from the cited reports. Unfortunately, in the rat preclinical report, ${ }^{7}$ after the second dose of PROG at $6 \mathrm{~h}$, no samples were taken up to $24 \mathrm{~h}$; thus, the extrapolation at the $12-\mathrm{h}$ time-point is our theoretical estimate (shown in broken lines).

The second implication of the preclinical studies is that the duration of dosing may have been too short in all of the Phase II and III clinical trials. Since prolonging treatment in rats from 3 to 5 days clearly improved several indices of progesterone efficacy (and might still not be long enough for optimal efficacy in rats), it is likely that the dosing duration needed to obtain optimal functional benefit in humans is substantially longer than 5 days. It is known that rats heal much more rapidly from brain injuries than humans: the recovery period for the brain injury caused by stroke (and presumably TBI) lasts about 1 month in rats, yet requires 3 months or more in humans. ${ }^{20}$ This is not surprising when one considers that 1 month of life for a rat is estimated to be equivalent to 2.5 human years. ${ }^{21,22}$ More rapid healing in rats than in humans also is consistent with the allometric scaling considerations used to adjust $\mathrm{mg} / \mathrm{kg}$ doses between humans and rats, which are based on the fact that the larger the animal species, the lower the specific (per gram) general metabolic rate ${ }^{16}$; thus, brain healing, like drug pharmacokinetics, would be expected to be more rapid in rats than in humans. In fact, it has been noted that protein turnover and metabolic rates are respectively and approximately $10 \times$ and $6 \times$ higher in rats than in humans, so appropriate scaling can be a critical factor in clinical trial designs. ${ }^{23,24}$ While faster rat drug metabolism translates into a need for higher $\mathrm{mg} / \mathrm{kg} /$ day drug doses for rats than humans, slower human general metabolism implies that drug presence needs to be more prolonged to facilitate the slower neural repair and remodeling processes following human central nervous system trauma. This would be particularly true for a drug like progesterone, which not only acts to reduce acute damage, but also promotes the later steps of neural regeneration and repair. ${ }^{11,12,18,25-27}$

\section{Conclusions}

Based on the results of preclinical progesterone TBI (and stroke) studies reported since the ProTECT II study was designed, and on the results of both Phase II and Phase III TBI clinical trials, we now propose that, in addition to the potential issues outlined by the authors of the Phase III trials, the two similarly designed Phase III trials may well have had negative outcomes for three other important reasons: 1) the significantly different doses and dosing durations used in the Protect II and Chinese Phase II trials may all have been suboptimal; 2) no Phase IIB trials were done to reconcile the two Phase II study protocols and their significantly different results, in order to optimize the clinical dose and schedule before moving into Phase III; and 3) both Phase III trials were designed similarly to ProTECT II (12 mg/ $\mathrm{kg}$ /day $\times 3$ or 5 days), without incorporating the new preclinical rat and Chinese Phase II results, as well as allometric scaling principles, into their design. Thus, on the one hand, and in hindsight, it is not surprising that, as in ProTECT II, no functional improvements were seen in the main study subpopulations of seriously injured patients in either Phase III study. On the other hand, the reason(s) for the lack of functional improvement in moderately-injured patients and improved mortality as reported in ProTECT II are unclear.

We hope that this new information provides a clear strategic rationale for a return to Phase II to determine whether progesterone is 
really a safe and effective neuroprotective agent for use in both male and female patients with brain injuries. Such a trial should examine the variables of both dose and duration of treatment, and also make use of functional indexes more precise and relevant to measurement of quantitative recovery than the GOS-E, DRS, and FIM. ${ }^{28-30}$

We advocate a study in which an empirically guided, adaptive dose and schedule optimization is performed. One plausible scenario would be to dose progesterone intravenously in parallel groups at 2 and $4 \mathrm{mg} / \mathrm{kg} / \mathrm{day}$, with each dose level subdivided into two longer dosing durations of perhaps 14 and 28 days, followed by a 1- to 2-day tapering of the dose as in Protect III. Patients could be followed monthly for the first 3 months to evaluate the kinetics of relative treated versus control healing during the primary 3-month human healing period; then at 6,9 , and 12 months to determine longer-term clinical tendencies. Finally, should this trial show clear efficacy and indicate an optimal dose/schedule combination such that no additional dose levels or schedules need be tested, we propose that one additional group be introduced, either in an adaptive design Phase II or in Phase III, in which a subgroup receives oral micronized progesterone (Prometrium ${ }^{\circledR}$; Catalent Pharma Solutions, St. Petersburg, FL) at modest doses giving PDLs of $\sim 25-50 \mathrm{ng} / \mathrm{mL}$ (the approximate PDLs accompanying efficacy in rat preclinical experiments), ${ }^{7}$ beginning at the end of the optimized intravenous infusion period/ dose combination and extending out to 3 months. This would make it possible to determine whether the continued presence of moderately elevated blood and brain concentrations of progesterone over the duration of the primary healing period would help to facilitate even better functional and morphological recovery than the optimal intravenous dosing protocol we are advocating for the Phase IIB studies.

\section{Author Disclosure Statement}

For R.B.H., no competing financial interests exist. Although D.G.S. and I.S. do not receive any financial gains, royalties, or licensing agreements from research on progesterone, they and Emory University currently hold patents related to the use of progesterone in TBI and certain forms of CNS tumors.

\section{References}

1. Wright, D.W., Yeatts, S.D., Silbergleit, R., Palesch, Y.Y., Hertzberg, V.S Frankel, M., Goldstein, F.C., Caveney, A.F., Howlett-Smith, H., Bengelink, E.M., Manley, G.T., Merck, L.H., Janis, L.S., and Barsan, W.G.; NETT Investigators. (2014). Very early administration of progesterone for acute traumatic brain injury. N. Engl. J. Med. 371, 2457-2466.

2. Skolnick, B.E., Maas, A.I., Narayan, R.K., van der Hoop, R.G., MacAllister, T., Ward, J.D., Nelson, N.R., and Stocchetti, N.; SYNAPSE Trial Investigators. (2014). A clinical trial of progesterone for severe traumatic brain injury. N. Engl. J. Med. 371, 2467-2476.

3. Wright, D.W., Kellermann, A.L., Hertzberg, V.S., Clark, P.L., Frankel, M., Goldstein, F.C., Salomone, J.P., Dent, L.L., Harris, O.A., Ander, D.S., Lowery, D.W., Patel, M.M., Denson, D.D., Gordon, A.B., Wald, M.M., Gupta, S., Hoffman, S.W., and Stein, D.G. (2007). ProTECT: A randomized clinical trial of progesterone for acute traumatic brain injury. Ann. Emerg. Med. 49, 391-402.

4. Xiao, G., Wei, J., Yan, W., Wang, W., and Lu, Z. (2008). Improved outcomes from the administration of progesterone for patients with acute severe traumatic brain injury: a randomized controlled trial. Crit. Care 12, R61.

5. Schwamm, L.H. (2014). Progesterone for traumatic brain injuryresisting the sirens' song. N. Engl. J. Med. 371, 2522-2523.

6. Wright, D.W., Ritchie, J.C., Mullins, R.E., Kellermann, A.L., and Denson, D.D. (2005). Steady-state serum concentrations of progesterone following continuous intravenous infusion in patients with acute moderate to severe traumatic brain injury. J. Clin. Pharmacol. 45, 640-648.

7. Wright, D.W., Bauer, M.E., Hoffman, S.W., and Stein, D.G. (2001) Serum progesterone levels correlate with decreased cerebral edema after traumatic brain injury in male rats. J. Neurotrauma 18, 901-909.
8. Shear, D.A., Galani, R., Hoffman, S.W., and Stein, D.G. (2002). Progesterone protects against necrotic damage and behavioral abnormalities caused by traumatic brain injury. Exp. Neurol. 178, 59-67.

9. Galani, R., Hoffman, S.W., and Stein, D.G. (2001). Effects of the duration of progesterone treatment on the resolution of cerebral edema induced by cortical contusions in rats. Restor. Neurol. Neurosci. 18, 161-166.

10. Goss, C.W., Hoffman, S.W., and Stein, D.G. (2003). Behavioral effects and anatomic correlates after brain injury: a progesterone doseresponse study. Pharmacol. Biochem. Behav. 76, 231-242.

11. Wali, B., Ishrat, T., Won, S., Stein, D.G., and Sayeed, I. (2014). Progesterone in experimental permanent stroke: a dose-response and therapeutic time-window study. Brain 137, 486-502.

12. Yousuf, S., Atif, F., Sayeed, I., Tang, H., and Stein, D.G. (2014). Progesterone in transient ischemic stroke: a dose-response study. Psychopharmacology (Berl) 231, 3313-3323.

13. Murphy, S.J., Traystman, R.J., Hurn, P.D., and Duckles, S.P. (2000). Progesterone exacerbates striatal stroke injury in progesterone- deficient female animals. Stroke 31, 1173-1178.

14. Reynolds, A.R. (2009). Potential relevance of bell-shaped and ushaped dose-responses for the therapeutic targeting of angiogenesis in cancer. Dose Response 8, 253-284.

15. McCord, J.M. (2008). Superoxide dismutase, lipid peroxidation, and bell-shaped dose response curves. Dose Response 6, 223-238.

16. Sharma, V. and McNeill, J.H. (2009). To scale or not to scale: the principles of dose extrapolation. Br. J. Pharmacol. 157, 907-921.

17. Wong, R., Ray, D., and Kendall, D.A. (2012). Progesterone pharmacokinetics in the mouse: implications for potential stroke therapy. J. Pharm. Pharmacol. 64, 1614-20.

18. Sayeed, I. and Stein, D.G. (2009). Progesterone as a neuroprotective factor in traumatic and ischemic brain injury. Prog. Brain. Res. 175, 219-237.

19. Stein, D.G. (2013). A clinical/translational perspective: Can a developmental hormone play a role in the treatment of traumatic brain injury? Horm. Behav. 63, 291-300.

20. Krakauer, J.W., Carmichael, S.T., Corbett, D., and Wittenberg, G.F. (2012). Getting neurorehabilitation right: what can be learned from animal models? Neurorehabil. Neural Repair 26, 923-931.

21. Sengupta, P. (2013). The laboratory rat: Relating its age with human's. Int. J. Prev. Med. 4, 624-630.

22. Quinn, R. (2005). Comparing rat's to human's age: how old is my rat in people years? Nutrition 21, 775-777.

23. Agoston, D.V. (2013). Of timescales, animal models, and human disease: The 50th anniversity of C. elegans as biological model. Front. Neurol. 4, 129.

24. Agoston, D.V. (2015). Bench-to-Bedside and bedside back to the bench; Seeking a better understanding of the acute pathophysiological process in severe Traumatic Brain Injury. Front. Neurol. 6, 47.

25. Schumacher, M., Hussain, R., Gago, N., Oudinet, J.P., Mattern, C., and Ghoumari, A.M. (2012). Progesterone synthesis in the nervous system: implications for myelination and myelin repair. Front. Neurosci. 6, 10.

26. De Nicola, A.F., Labombarda, F., Deniselle, M.C., Gonzalez, S.L., Garay, L., Meyer, M., Gargiulo, G., Guennoun, R., and Schumacher, M. (2009). Progesterone neuroprotection in traumatic CNS injury and motoneuron degeneration. Front. Neuroendocrinol. 30, 173-187.

27. Wei, J. and Xiao, G.M. (2013). The neuroprotective effects of progesterone on traumatic brain injury: current status and future prospects. Acta Pharmacologica Sinica 34, 1485-1490.

28. Poon, W., Vos, P., Muresanu, D., Vester, J., von Wild, K., Homberg, V., Wang, E., Lee, T.M., and Matula, C. (2015). Cerebrolysin asian pacific trial in acute brain injury and neurorecovery: design and methods. J. Neurotrauma 32, 571-580.

29. Maas, A.I., Menon, D.K., Lingsma, H.F., Pineda, J.A., Sandel, M.E., and Manley, G.T. (2012). Re-orientation of clinical research in traumatic brain injury: report of an international workshop on comparative effectiveness research. J. Neurotrauma 29, 32-46.

30. Stein, D.G. (2015). Embracing failure: What the Phase III progesterone studies can teach us about TBI clinical trials. Brain Injury. 2015 Aug 14; [Epub ahead of print].

Address correspondence to:

Donald G. Stein, PhD

Emory University

1365 B Clifton Road NE, Suite 5100

Atlanta, GA 30322

E-mail: dstei04@emory.edu 